\title{
Just the facts: how to diagnose and manage patients with multiple myeloma in the emergency department?
}

\author{
Arleigh McCurdy ${ }^{1,2} \cdot$ Hira Mian $^{3} \cdot$ Hans Rosenberg ${ }^{1,2}[$ \\ Received: 23 October 2021 / Accepted: 14 January 2022 / Published online: 18 February 2022 \\ (c) The Author(s), under exclusive licence to Canadian Association of Emergency Physicians (CAEP)/ Association Canadienne de Médecine d'Urgence \\ (ACMU) 2022
}

Keywords Multiple myeloma $\cdot$ Hematology $\cdot$ Emergency medicine

\section{Clinical scenario}

A 67-year-old male presents to a rural emergency department (ED) with a 2-month history of progressive fatigue and weight loss. He also noted significant back pain in the preceding 2 weeks. His heart rate is 110 beats per minute, the rest of his vital signs are within normal limits. He has skin pallor and tenderness to percussion in the mid-thoracic spine (Fig. 1).

\section{What is multiple myeloma and which patients are typically affected?}

Multiple myeloma is a hematologic malignancy characterized by the clonal proliferation of plasma cells in the bone marrow. It is the second most common hematologic malignancy, affecting approximately 3300 patients in Canada annually. Multiple myeloma is typically a disease of older adults, with median age at diagnosis of 65-70 years [1]. However, multiple myeloma also occurs in younger adults, with roughly $16 \%$ of cases occurring in patients younger than 50 years [2]. It is more common in males and black patients. Multiple myeloma remains non-curable with multiple episodes of treatment and disease relapse. The median survival of multiple myeloma patients is approximately 5-10 years.

Hans Rosenberg

hrosenberg@toh.ca

1 The Ottawa Hospital, Ottawa, ON, Canada

2 University of Ottawa, Ottawa, ON, Canada

3 Juravinski Cancer Centre, Hamilton, ON, Canada

\section{How do patients with multiple myeloma present?}

Multiple myeloma patients present commonly with one or more of the "CRAB" symptoms which reflect end organ damage: hyperCalcemia, Renal failure, Anemia, or lytic Bone lesions. Other common symptoms include fatigue, weight loss, and infections. Symptoms of hypercalcemia, renal failure and anemia may be challenging to discern. Bone pain is present in $60 \%$ of patients at the time of diagnosis, and $80 \%$ of patients have osteolytic bone lesions on $\mathrm{x}$-ray [3]. Symptoms range from asymptomatic to severe bone pain from acute fractures, as well as neurologic compromise from spinal cord compression.

\section{What investigations are required to expedite the diagnosis and evaluate for complications?}

If multiple myeloma is suspected clinically, basic investigations should include a complete blood count, electrolytes, creatinine, albumin, total protein, calcium, quantitative immunoglobulins (IgA, IgG and IgM levels), and plain films of symptomatic bone areas. Investigation to look for the presence of a monoclonal protein should be ordered if possible, including serum and urine protein electrophoresis and immunofixation and a serum free light chain assay. Multiple myeloma would classically reveal a high total protein and low serum albumin, and this combination should raise the suspicion.

\section{Are there emergencies that the ED physicians should be on the lookout for in patients with multiple myeloma?}

The most common serious emergencies in patients with multiple myeloma are spinal cord compression, acute kidney injury 


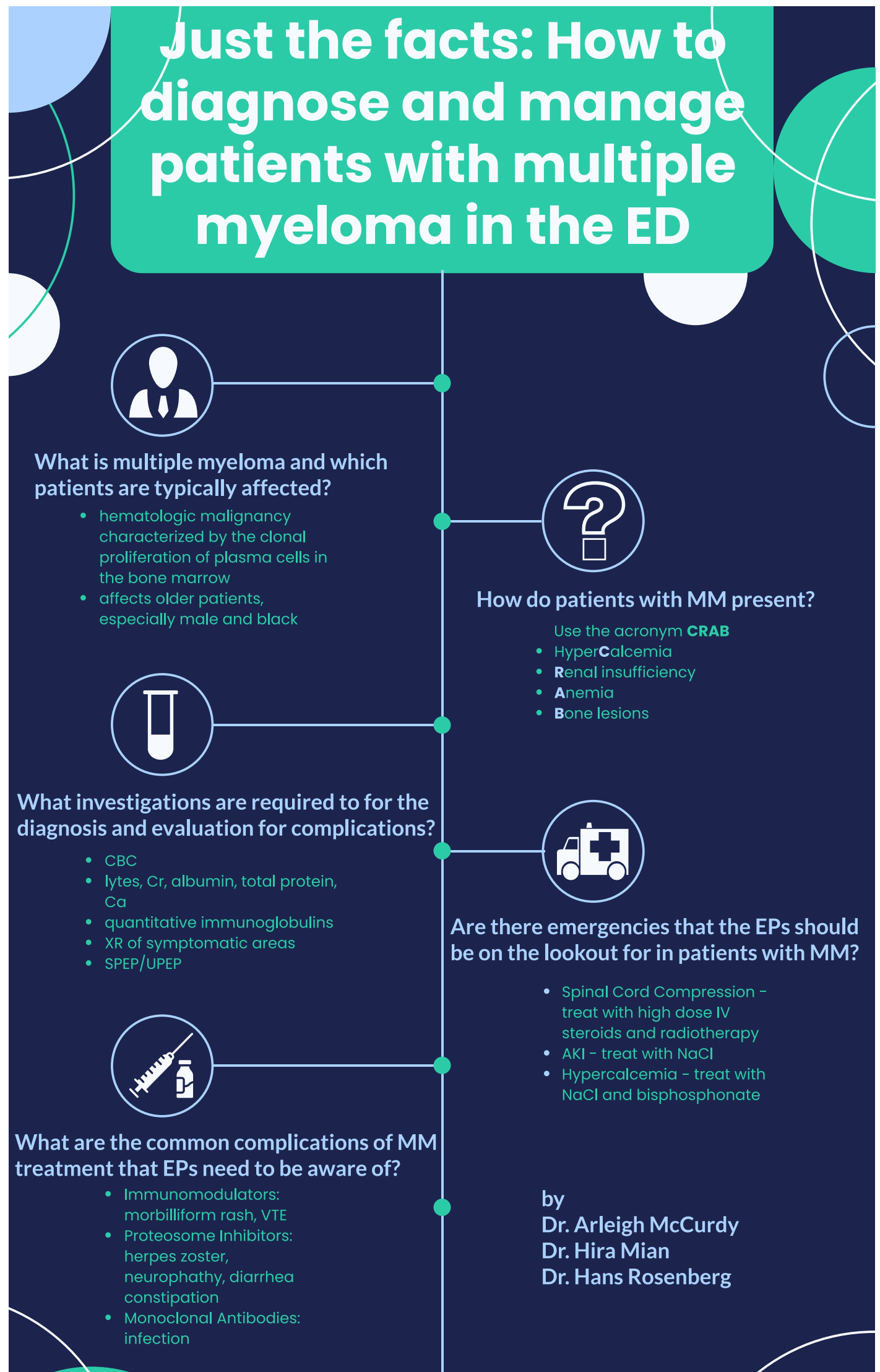

Fig. 1 Infographic-How to diagnose and manage patients with multiple myeloma in the ED 
and hypercalcemia. Spinal cord compression can present with back pain, lower extremity paresthesia and/or weakness/paralysis, ataxia. Cauda equina syndrome can be seen with bowel and/or bladder dysfunction (usually urinary retention or fecal incontinence), or saddle anaesthesia. The upper extremities can be involved in thoracic or cervical spine lesions. Magnetic resonance imaging of the entire spine should be performed immediately if spinal cord compression is suspected, computed tomography is an alternative if magnetic resonance imaging is not available [4]. If spinal cord compression is confirmed, treatment needs to begin as soon possible to prevent permanent neurologic sequelae. Multiple myeloma patients seen in community/rural ED with features concerning for spinal cord compression should be transferred for necessary imaging and emergent treatment. Urgent management of spinal cord compression in multiple myeloma includes high dose intravenous steroids [4] (i.e., dexamethasone $10 \mathrm{mg}$ intravenous every $6 \mathrm{~h}$ ); however, consultation with radiation oncology and/or neurosurgery is required for further management.

Acute kidney injury (usually due to accumulation of light chains secreted by multiple myeloma cells) occurs in up to $50 \%$ of patients with multiple myeloma, and can be the initial presentation, occur at relapse, or as a treatment complication [5]. Management should include optimizing hemodynamics, brisk hydration with saline to establish high urine flow, and correction of precipitating factors which could include hypercalcemia [5].

Hypercalcemia occurs in roughly $15-30 \%$ of patients at the time of diagnosis [5], but it is more common if the patient also has renal failure. Treatment requires aggressive hydration in combination with intravenous bisphosphonate, dose-adjusted to estimated glomerular filtration rate. Historically diuretics were recommended, however they should only be used in the event of concomitant severe volume overload.

\section{What are the common complications of multiple myeloma treatment that ED physicians need to be aware of?}

The three main classes of drugs used to treat myeloma are the immunomodulators (lenalidomide, pomalidomide), Proteosome inhibitors (bortezomib, carfilzomib), and monoclonal antibodies (daratumumab). Immunomodulators can cause a classic morbilliform rash in which typically involves the trunk and proximal limbs. Mild to moderate cases are managed with topical corticosteroids and antihistamines, whereas drug interruption and systemic steroids are reserved for severe reactions. Immunomodulators also carry a risk of venous thromboembolism, occurring in $5-20 \%$ of multiple myeloma patients [6]. Proteosome inhibitors carry a significant risk of herpes zoster reactivation, especially if the patient is not taking prophylactic antivirals. Peripheral neuropathy, diarrhea and constipation are also common side effects of proteosome inhibitors. Carfilzomib in particular has been associated with heart failure in rare circumstances. Infections are common in patients with myeloma due to the immune dysregulation of the illness and treatment. Monoclonal antibodies exacerbate the risk of infection and most notably pneumonia, with upwards of $80 \%$ of monoclonal antibodies treated patients developing infections, of which up to $20 \%$ are severe [7]. Additionally, many multiple myeloma drugs can cause cytopenias and therefore transfusions and/ or urgent management of infections with febrile neutropenia protocol may be required.

\section{Case resolution}

The patient's blood work shows a hemoglobin of $97 \mathrm{~g} / \mathrm{L}$, creatinine of $232 \mathrm{micromol} / \mathrm{L}$, calcium of $2.8 \mathrm{mmol} / \mathrm{L}$ with an albumin of $26 \mathrm{~g} / \mathrm{L}$. Thoracic and lumbar spine radiograph shows multiple lytic lesions with a fracture at the 12th thoracic vertebra. He has no neurological signs on exam and a post void residual of $20 \mathrm{~mL}$. Given the findings the ED physician is highly suspicious for multiple myeloma as a diagnosis. She begins immediate fluid hydration with normal saline and pamidronate. The regional hematology consultant is contacted and requests for the patient to be transferred to the nearby academic centre for admission.

\section{Declarations}

Conflict of interest All three authors do not have any conflicts of interest to declare.

\section{References}

1. Public Health Agency of Canada; Statistics Canada; Canadian Cancer Society. Release notice-Canadian cancer statistics. Health Promot Chronic Dis Prev Can. 2019;39:255.

2. Ludwig H, Bolejack V, Crowley J, et al. Survival and years of life lost in different age cohorts of patients with multiple myeloma. J Clin Oncol. 2010;28:1599-605.

3. Kyle RA, Gertz MA, Witzig TE, et al. Review of 1027 patients with newly diagnosed multiple myeloma. Mayo Clin Proc. 2003;78:21-33.

4. Chen B, Cai L, Zhou F. Management of acute spinal cord compression in multiple myeloma. Crit Rev Oncol Hematol. 2021;160:103205.

5. Kyle RA, Rajkumar SV. Treatment of multiple myeloma: a comprehensive review. Clin Lymph Myeloma. 2009;9:278-88.

6. Swan D, Rocci A, Bradbury C, Thachil J. Venous thromboembolism in multiple myeloma-choice of prophylaxis, role of direct oral anticoagulants and special considerations. Br J Haematol. 2018;183:538-56.

7. Vitkon R, Netanely D, Levi S, et al. Daratumumab in combination with proteasome inhibitors, rapidly decreases polyclonal immunoglobulins and increases infection risk among relapsed multiple myeloma patients: a single center retrospective study. Ther Adv Hematol. 2021. https://doi.org/10.1177/20406207211035272. 